\title{
STUDY OF TWO ROBUST CONTROLS FOR AN HYDRAULIC ACTUATOR
}

\author{
Valérie POMMIER ${ }^{\ddagger}$, Roland MUSSET ${ }^{*}$, Patrick LANUSSE ${ }^{\dagger}$ and Alain OUSTALOUP ${ }^{\dagger}$ \\ ENSICA - 1, Place Blouin, 31056 Toulouse - France \\ fax: +33 (0)561 618686 - e-mail: valerie.pommier@ensica.fr \\ *CRAN-ACS - 186, rue de Lorraine, 54400 Longwy -France \\ fax: +33 (0)382 396227 - e-mail: musset@iut-longwy.uhp-nancy.fr \\ †LAP UMR 5131 CNRS/Université Bordeaux I/ENSEIRB \\ 351, Cours de la Libération, 33405 Talence - France \\ fax: +33 (0)556 842417 - e-mail: lanusse@lap.u-bordeaux.fr
}

Key words: Robust control, CRONE Control, H infinity control, $\mu$-analysis, hydraulic actuator

\begin{abstract}
Two robust control design methodologies are analyzed and compared in this article: the $\mathrm{H}$-infinity control system design and the CRONE control system design. The aim of this article is to give practical considerations that will help a designer to choose between these two methodologies. The example of an electrohydraulic actuator is given in order to evaluate the implementation of each methodology and to compare the final performance.
\end{abstract}

\section{1 - Introduction}

The importance of robust control is growing in order to ensure good performance despite the imprecision of the model and the parameters variation. Several robust methodologies are accessible to the designer. Each methodology offers advantages and drawbacks and it is not always easy to choose the right methodology regarding the process to control. In this paper, two robust control methodologies are analyzed and compared to help the designer in its choice. Both methodologies are frequencydomain based methodologies, so they required the same basic understanding on control methodology.

The article only deals with SISO systems. It first introduces the two methodologies under study: the H-infinity control design and the CRONE control design. Both methodologies use the common unity feedback configuration (Figure 1). Then these methodologies are applied to an electro-hydraulic actuator and performance is compared.

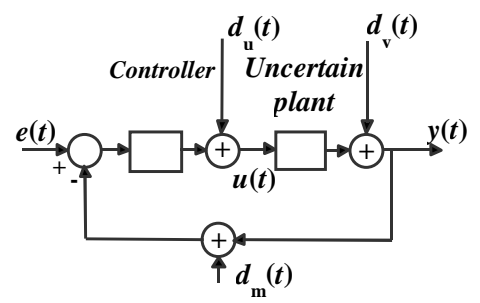

Figure. 1 - Common unity feedback configuration

\section{2 - H-infinity control-system design}

H-infinity loop shaping was proposed by McFarlane and Glover [3] in the late eighties. Since then, much work has been done to develop H-infinity loop shaping. H-infinity control is well-known and so the principles will be recalled briefly.

Let's consider the generalized block diagram shown in Figure 2. The plant $P$ is assumed to be linear and time-invariant. The general control problem it to synthesize a controller that will keep the size of the performance variables $z$ small in the presence of the exogenous signals $w$.

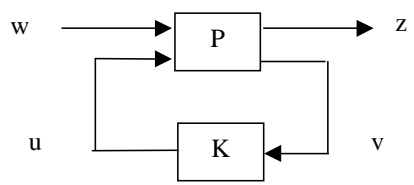

Figure 2 - General control configuration If $P(\mathrm{~s})$ is written :

$$
P(s)=\left(\begin{array}{cc}
P_{z w}(s) & P_{z u}(s) \\
P_{v w}(s) & P_{v u}(s)
\end{array}\right),
$$

the matrix between $w$ and $z$ called Linear Fractional Transformation is:

$$
\begin{aligned}
& Z(s)=F(P(s), K(s)) W(s) \\
& =\left[P_{z w}(s)+P_{z u}(s) K(s)\left(I-P_{v u}(s) K(s)\right)^{-1} P_{v w}(s)\right] W(s)
\end{aligned}
$$

and so the problem to be solved is: $P(\mathrm{~s})$ and $\gamma$ being given, find a stabilizing controller $K(\mathrm{~s})$ such that $\|F(P(s), K(s))\|_{\infty}<\gamma$. This problem can be solve by two methods: by Ricatti equations or by Linear Matrix Inequalities $[1,2,4]$.

It is often said that H-infinity control approach leads to a closed-loop system with good robustness properties [4]. The foundation of this widespread idea is the well-known Small Gain Theorem saying that a sufficient condition for stability of the closed-loop system is that $\left\|T_{z w}\right\|_{\infty}\|\Delta\|_{\infty}<1$, where $\Delta$ is the system uncertainty model. This leads to the stability robustness shown in Figure 3. 


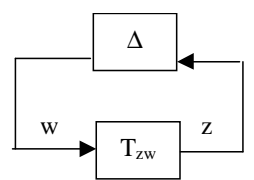

Figure 3 - Stability robustness

In order to improve the H-infinity design methodology, the designer may add the desired control system performance and robustness to the previous problem. This can be done through the choice of the plant $P$, that can contain both the system model $G$ and the design weighting functions $W i$ that permit to fit more precisely the sensitivity functions. The resulting model is called "augmented plant". In this article, the proposed configuration is shown in Figure 4. It contains three weighting functions.

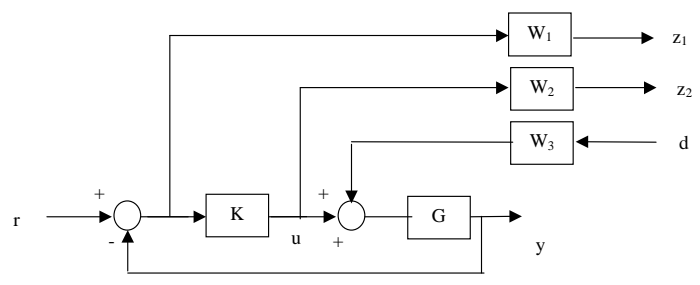

Figure 4 - Design configuration

Considering $r$ and $d$ as inputs and $z 1$ and $z 2$ as outputs, the matrix representation of this configuration is written:

$$
\left(\begin{array}{c}
z_{1}(s) \\
z_{2}(s)
\end{array}\right)=\left(\begin{array}{cc}
W_{1} S(s) & W_{1} S G W_{3}(s) \\
W_{2} K S(s) & W_{2} T W_{3}(s)
\end{array}\right)\left(\begin{array}{c}
R(s) \\
D(s)
\end{array}\right) .
$$

with $S=\frac{1}{1+K G}$ and $T=\frac{K G}{1+K G}$.

and now the problem to be solved is: $\gamma$ being given, find a stabilizing controller $K(\mathrm{~s})$ that minimizes:

$$
\left\|\left(\begin{array}{cc}
W_{1} S(s) & W_{1} S G W_{3}(s) \\
W_{2} K S(s) & W_{2} T W_{3}(s)
\end{array}\right)\right\|_{\infty}<\gamma \leq 1 .
$$

From relation (3), you can deduce that the robustness is warranted if:

$$
\|\Delta\|_{\infty}<\left\|W_{2}\right\|_{\infty} \text { if } \Delta \text { is additive, id } \Delta=G-G_{\text {nom }} \text { where } \mathrm{G}
$$

is the set of uncertain plants and Gnom the nominal plant (Figure 5a).

- $\|\Delta\|_{\infty}<\left\|W_{2} W_{3}\right\|_{\infty}$ if $\Delta \quad$ is multiplicative, id $\Delta=\left(G-G_{\text {nom }}\right) G_{\text {nom }}^{-1}$ (Figure 5b).

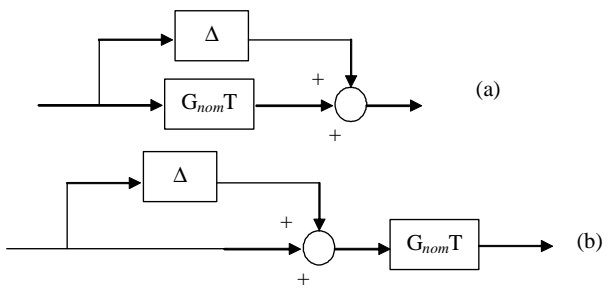

Figure 5 - Unstructured uncertainty (a) additive (b) multiplicative

Note that the uncertainty defined above is said "unstructured".

\section{3 - CRONE control-system design}

CRONE (the French acronym of "Commande Robuste d'Ordre Non Entier") control system design [6,7] is a frequency-domain based methodology, using complex fractional differentiation. It permits the robust control of perturbed linear plants using the common unity feedback configuration. It consists on determining the nominal and optimal open-loop transfer function that guaranties the required specifications. This methodology uses fractional derivative orders (real or complex) as high level parameters that make you easy the design and optimization of the control-system. While taking into account the plant right half-plane zeros and poles, the controller is then obtained from the ratio of the open-loop frequency response to the nominal plant frequency response. Three Crone control generations have been developed, successively extending the application fields. In this paper, only the third generation is introduced.

The initial third generation Crone method is based on a particular Nichols locus called a generalized template and defined by an any-direction straight line segment around open-loop gain cross-over frequency $\omega$ cg (Figure 6). This generalized template is based on the real part (with respect to imaginary unit i) of complex fractional integration:

$$
\beta(s)=\left[\cosh \left(b \frac{\pi}{2}\right)\right]^{-1} \mathfrak{R}_{/ \mathrm{i}}\left[\left(\frac{\omega_{\mathrm{cg}}}{s}\right)^{n}\right],
$$

with $n=a+\mathrm{i} b \in \mathrm{C}_{\mathrm{i}}$ and $s=\sigma+\mathrm{j} \omega \in \mathrm{C}_{\mathrm{j}}$.

In the Nichols chart at frequency $\omega \mathrm{cg}$, the real order $a$ determines the phase placement of the template, and then the imaginary order $b$ determines its angle to the vertical.

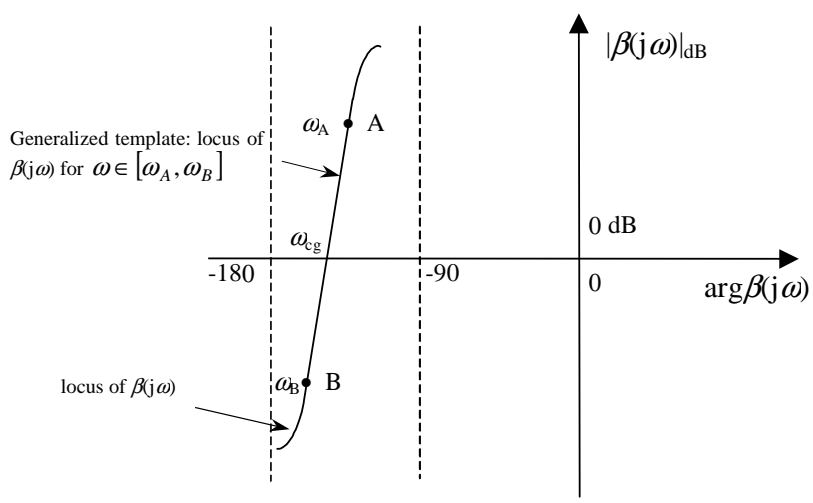

Figure 6 - Representation in the Nichols chart of the generalized template by an any-direction straight line

In the version of third generation Crone control design used in this article, the open-loop transfer function defined for the nominal state of the plant, $\beta_{n} \mathrm{om}(s)$, takes into account the control specifications at low and high frequencies and a set of band-limited generalized templates around resonant frequency $\omega \mathrm{r}$. Thus $\beta_{n} \mathrm{om}(s)$ is defined by: 


$$
\begin{aligned}
& \beta_{\text {nom }}(s)=K\left(\frac{\omega_{-N^{-}}}{s}+1\right)^{n_{1}} \prod_{-N^{+}}^{N^{+}}\left(\frac{1+s / \omega_{k+1}}{1+s / \omega_{k}}\right)^{a_{k}} * \\
& \left(\Re_{/ \mathrm{i}}\left[\left(C_{k} \frac{1+s / \omega_{k+1}}{1+s / \omega_{k}}\right)^{\mathrm{i} b_{k}}\right]\right)^{-\operatorname{sign}\left(b_{k}\right)} \frac{1}{\left(1+s / \omega_{N^{+}+1}\right)^{n_{\mathrm{h}}}}
\end{aligned}
$$

$$
\begin{aligned}
& \text { where } \\
& \begin{aligned}
C_{0} & =\left[\left(1+\omega_{\mathrm{r}}^{2} / \omega_{0}^{2}\right) /\left(1+\omega_{\mathrm{r}}^{2} / \omega_{1}^{2}\right)\right]^{1 / 2} \text { and } \\
C_{k} & =\left[\omega_{k+1} / \omega_{k}\right]^{1 / 2} \text { for } \mathrm{k} \neq 0
\end{aligned}
\end{aligned}
$$

$K$ ensures a gain of $0 \mathrm{~dB}$ at $\omega \mathrm{cg}$, the order $n \mathrm{l}$ fixes the steady state behavior of the closed-loop system at low frequencies, and the value of $n_{\mathrm{h}}$ has to be chosen as equal to or greater than the high-frequency order of the plant.

Once the transfer function is defined, the third generation CRONE methodology can be described in five points:

1 - You determine the nominal plant transfer function and the uncertainty domains. For a given frequency, an uncertainty domain (called "template" by the QFT users) is the smallest hull including the possible frequency responses of the plant. The use of the edge of the domains permits to take into account the uncertainty with the smallest number of data. To construct this domain securely, the simplest way is to define it convexly.

2 - You specify some parameters of the open-loop transfer function defined for the nominal state of the plant: the number of band-limited generalized templates $N^{+}$and $N$, and the rational orders $n \mathrm{l}$ and $n_{\mathrm{h}}$.

3 - You specify the bounds of the sensibility functions that you would like to obtain. Let $M_{r_{\text {nom }}}$ be the required resonant peak of the nominal complementary sensitivity function.

4 - Using the nominal plant locus and the uncertainty domains in the Nichols chart, you optimize the parameters $w \mathrm{r}, a \mathrm{k}$ and $b \mathrm{k}$ (for $\mathrm{k} \neq 0$ ), $w \mathrm{k}$ and $w \mathrm{k}+1$ in order to obtain the optimal open-loop Nichols locus. An open-loop Nichols locus is defined as optimal if it tangents the $M_{r_{\text {nom }}}$ magnitude contour and if it minimizes the variations of $M_{r}$ for the other parametric states. By minimizing the cost function $J=\left(M_{r_{\max }}-M_{r_{\text {nom }}}\right)^{2}$ where $M_{r_{\max }}$ is the maximal value of resonant peaks $M_{r}$, the optimal open-loop Nichols locus positions the uncertainty domains correctly, so that they overlap the low stability margin areas as little as possible (Figure 7: case (c) is the best configuration). The minimization of $J$ is carried out under a set of shaping constraints on the four usual sensitivity functions.
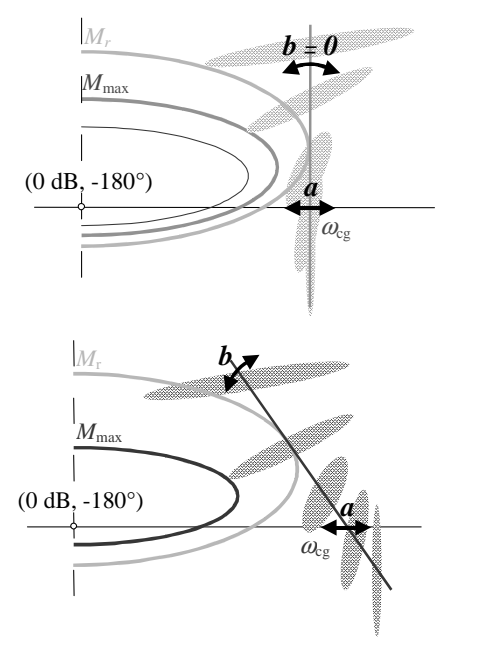

(b)

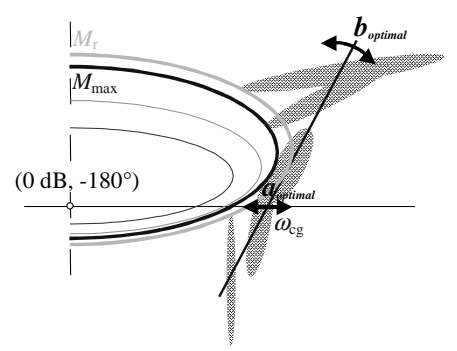

(c)

Figure 7 - Optimal open-loop Nichols locus to position the uncertainty domains

5 - The last point is the synthesis of the controller. While taking into account the plant right half-plane zeros and poles, the controller is deduced by frequency-domain system identification of the ratio of $\beta_{\text {nom }}(\mathrm{j} \omega)$ to the nominal plant function transfer $G_{\text {nom }}(\mathrm{j} \omega)$. The resulting controller $K(s)$ is a rational transfer function.

All the five points can be achieved using the CRONE Toolbox developed by the CRONE Team [8].

\section{4 - Application to an electrohydraulic actuator}

\section{1 - Description of the system}

The system under study is an electrohydraulic actuator linked to an uncertain load described by a mass-damper-spring set (figure 8). The viscous coefficient $b \mathrm{~s}$ is supposed to be constant, the mass $M \mathrm{~s}$ and the stiffness $K \mathrm{~s}$ are supposed to vary slowly (compared with the system dynamics). The values of these parameters are:

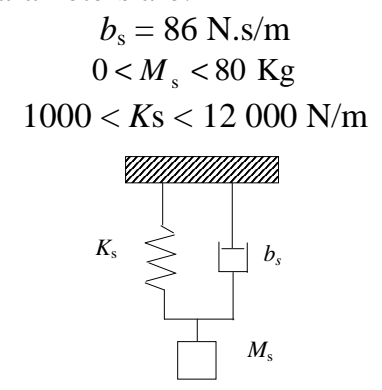

Figure 8 - Model of the load 
The complete model of the electrohydraulic test bench is given by table 1 and by the following nonlinear state-space model:

$$
\left\{\begin{array}{l}
\dot{X}=f_{1}(X)+g(X) u \text { if } y_{\mathrm{u}} \geq 0 \\
\dot{X}=f_{2}(X)+g(X) u \text { if } y_{\mathrm{u}}<0 \\
Y=h(X)
\end{array}\right.
$$

with: $X^{T}=\left[\begin{array}{lllll}P_{1} & P_{2} & v & y & y_{\mathrm{u}}\end{array}\right]$,

$$
\begin{aligned}
& f_{1}(X)=\left(\begin{array}{c}
\frac{B}{V_{0}+S_{0} y}\left(\begin{array}{l}
-S_{0} v+y_{\mathrm{u}} \sqrt{\left|P_{\mathrm{s}}-P_{1}\right|} \operatorname{sign}\left(P_{\mathrm{s}}-P_{1}\right) \\
-\lambda\left(P_{1}-P_{2}\right)-\lambda_{\mathrm{p}}\left(P_{1}-P_{\mathrm{P}_{1}}\right)
\end{array}\right) \\
\frac{B}{V_{0}-S_{0} y}\left(\begin{array}{l}
S_{0} v-y_{\mathrm{u}} \sqrt{\mid P_{2}-P_{\mathrm{r}}} \mid \operatorname{sign}\left(P_{2}-P_{\mathrm{r}}\right) \\
-\lambda\left(P_{1}-P_{2}\right)-\lambda_{\mathrm{p}}\left(P_{2}-P_{\mathrm{P} 2}\right)
\end{array}\right) \\
\frac{1}{M_{\mathrm{s}}+M_{0}}\left(S_{0} P_{1}-S_{0} P_{2}-K_{\mathrm{s}}\left(y-y_{0}\right)-b_{\mathrm{s}} v-F_{\mathrm{f}}\right) \\
v \\
-\omega_{\mathrm{a}}
\end{array}\right), \\
& f_{2}(X)=\left(\begin{array}{c}
\frac{B}{V_{0}+S_{0} y}\left(\begin{array}{c}
-S_{0} v+y_{\mathrm{u}} \sqrt{\left|P_{1}-P_{r}\right|} \operatorname{sign}\left(P_{1}-P_{r}\right) \\
-\lambda\left(P_{1}-P_{2}\right)-\lambda_{\mathrm{p}}\left(P_{1}-P_{\mathrm{P}_{1}}\right)
\end{array}\right) \\
\frac{B}{V_{0}-S_{0} y}\left(\begin{array}{l}
S_{0} v-y_{\mathrm{u}} \sqrt{\left|P_{s}-P_{2}\right|} \operatorname{sign}\left(P_{s}-P_{2}\right) \\
-\lambda\left(P_{1}-P_{2}\right)-\lambda_{\mathrm{p}}\left(P_{2}-P_{\mathrm{P} 2}\right)
\end{array}\right) \\
\frac{1}{M_{\mathrm{s}}+M_{0}}\left(S_{0} P_{1}-S_{0} P_{2}-K_{\mathrm{s}}\left(y-y_{0}\right)-b_{s} v-F_{\mathrm{f}}\right) \\
v \\
-\omega_{a}
\end{array}\right),
\end{aligned}
$$$$
g^{T}(X)=\left(\begin{array}{lllll}
0 & 0 & 0 & 0 & k_{\mathrm{u}} \omega_{\mathrm{a}}
\end{array}\right) \text { and } h(X)=v .
$$

\begin{tabular}{|c|c|c|}
\hline $\mathrm{P}_{\mathrm{s}}$ & supply pressure & 240 bar \\
\hline $\mathrm{P}_{\mathrm{r}}$ & tank pressure & $7.5 \mathrm{bar}$ \\
\hline $\mathrm{P}_{1}, \mathrm{P}_{2}$ & cylinder chamber pressures & bar \\
\hline $\mathrm{P}_{\mathrm{P} 1}$ & hydrostatic bearings pressure & $236 \mathrm{bar}$ \\
\hline $\mathrm{P}_{\mathrm{P} 2}$ & hydrostatic bearings pressure & $212 \mathrm{bar}$ \\
\hline $\mathrm{B}$ & Bulk modulus & $10^{9} \mathrm{bar}$ \\
\hline $\mathrm{V}_{1}, \mathrm{~V}_{2}$ & cylinder chamber volumes & $\mathrm{m}^{3}$ \\
\hline $\mathrm{y}_{\mathrm{u}}$ & Amplification stage spool & $\mathrm{m}$ \\
\hline $\mathrm{V}_{0}$ & position & \\
\hline $\mathrm{M}_{0}$ & cylinder half-volume & $245.10^{-7} \mathrm{~m}^{3}$ \\
\hline $\mathrm{S}_{0}$ & cylinder rod effective area & $243.10^{-6} \mathrm{~m}^{2}$ \\
\hline $\mathrm{y}$ & cylinder rod position & $\mathrm{m}$ \\
\hline $\mathrm{V}$ & cylinder rod velocity & $\mathrm{m} / \mathrm{s}$ \\
\hline $\mathrm{k}_{\mathrm{u}}$ & $\begin{array}{c}\text { amplification stage gain } \\
\end{array}$ & $\begin{array}{c}1.17 * 10^{-6} \\
\mathrm{~m}^{3} / \mathrm{s} / \mathrm{A}\end{array}$ \\
\hline$\lambda$ & cylinder leakage coefficient & $1.10^{-11} \mathrm{~s}$ \\
\hline$\lambda_{\mathrm{p}}$ & $\begin{array}{c}\text { hydrostatic bearings leakage } \\
\text { coefficient }\end{array}$ & $0,5.10^{-12} \mathrm{~s}$ \\
\hline$\omega_{\mathrm{a}}$ & $\begin{array}{c}\text { cut-off frequency of the } \\
\text { amplification stage }\end{array}$ & $942 \mathrm{rad} / \mathrm{s}$ \\
\hline$F_{\mathrm{f}}$ & friction force & $\mathrm{N}$ \\
\hline
\end{tabular}

Table 1 - Notations for the electrohydraulic model

Note: The friction force $F_{\mathrm{f}}$ can be considered null thanks to the hydrostatic bearings.
As H-infinity control and CRONE control are to be applied on linear system, it is first necessary to linearize the model of the electrohydraulic actuator. To this end, an input-output linearization under diffeomorphism and feedback is achieved. So that this linearization is available whatever the load and its parameters, the output considered for the linearization is the pressure difference. Indeed, if this output is chosen, the linearization law does not depend on the parameters of the load. Moreover to get a relative degree equal to 1 in order to simplify the linearization and its numerization, the linear model of the amplification stage is not taken into account in the linearization. However it is necessary to consider afterwards an inverse band-limited model of this stage (see figure 9).

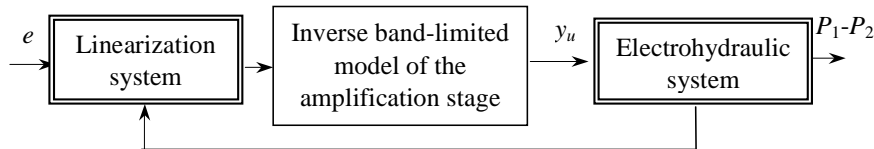

Figure 9 - Scheme of the linearization strategy

Finally the linearized model of the electrohydraulic system between input $e$ and output $(P 1-P 2)$ is described by:

$$
\frac{P_{1}(s)-P_{2}(s)}{E(s)}=k_{\mathrm{p}} /\left(\frac{s}{\alpha}+1\right)\left(\frac{s}{5000}+1\right)
$$

with $k_{\mathrm{p}}=7.108$ and $\alpha=2000$ chosen so that the input-output linearized system has the same behavior than the first-order linearized system around the operating point defined by $v=0$ and $y=0$ and $1 /(s+5000)$ coming from the band-limited model of the amplification stage.

Once the system is linearized, a robust control law is computed to control the velocity by using the new model:

$$
\left\{\begin{array}{l}
\dot{X}=f(X)+g(X) e \\
Y=h(X)
\end{array}\right.
$$

with: $X^{T}=\left[\begin{array}{llll}\Delta P & \Delta \dot{P} & v & y\end{array}\right]$ where $\quad \Delta P=P_{2}-P_{1}$

$$
\text { and } f(X)=\left(\begin{array}{c}
\Delta \dot{P} \\
-5000 \alpha \Delta P-(5000+\alpha) \Delta \dot{P} \\
\frac{1}{M_{\mathrm{s}}+M_{0}}\left(S \Delta P-K_{\mathrm{s}}\left(y-y_{0}\right)-b_{\mathrm{s}} v-F_{\text {frot }}\right) \\
v
\end{array}\right) \text {, }
$$

with $g^{T}(X)=\left(\begin{array}{llll}0 & 5000 \alpha \Delta P & 0 & 0\end{array}\right)$ and $h(X)=v$, this model being sampled at period $T_{s}=1 \mathrm{~ms}$.

Note: As the control-system is implemented numerically, and as H-infinity and CRONE design are continuous frequency approaches, the discrete-time design problem is transformed into a pseudo-continuous problem using the $z$ and bilinear $w$ transformation defined by :

$z^{-1}=\frac{1-w}{1+w} \quad$ with $\quad w=\mathrm{j} v$ and $\quad v=\tan \left(\frac{\omega T_{\mathrm{s}}}{2}\right)$. 


\section{2 - H-infinity controller}

First, the weighting functions have to be chosen regarding the following requirements:

$\S$ the control-system bandwidth: $v_{\mathrm{c}}=0.06$

$\$$ a modulus margin greater than 0.7

$\$$ the additive uncertainties and the multiplicative uncertainties.

Thus, it has been chosen (Figure 10):

$$
\begin{aligned}
& W_{1}(j v)=\frac{j v+5 e^{-2}}{1.3\left(j v+6 e^{-5}\right)}, \quad W_{2}(j v)=1 e^{-3} \frac{\frac{j v}{0.5}+1}{\frac{j v}{1 e^{4}}+1} \quad \text { and } \\
& W_{3}(j v)=1 e^{-4} \frac{\frac{j v}{0.001}+1}{\frac{j v}{10}+1}
\end{aligned}
$$

Then the controller is computed using the robust control Toolbox of Mathworks [5]:

$$
\begin{aligned}
& K(w)=-5.83 e^{-4} * \\
& \frac{\left(w+1 e^{4}\right)(w-15.98)(w+2.38)(w+0.75644)\left(w^{2}+8 e^{-4} w+2.065 e^{-5}\right)}{(w+126)(w+10)(w+3.19)(w+1.07)\left(w+6 e^{-5}\right) w}
\end{aligned}
$$
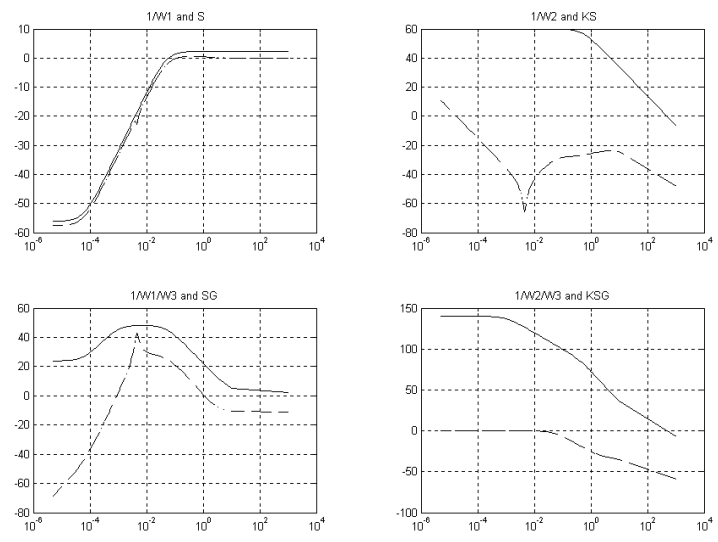

Figure 10 - Weighting functions (__ and closed-loop responses (- - )

\section{3 - CRONE controller}

You follow the methodology given in section 3 to compute the CRONE controller.

1 - You determine the nominal plant transfer function and the uncertainty domains (Figure 11).

2 - You specify some parameters of the open-loop transfer function defined for the nominal state of the plant:

the number of band-limited generalized templates: $N^{+}=1$ and $N^{-}=1$,

the order $n \mathrm{l}=1$ and the order $n_{\mathrm{h}}=3$.

3 - You specify the sensibility functions that you would like to obtain for the nominal plant. Let $M_{r_{n o m}}=1 \mathrm{~dB}$ be the required resonant peak of the nominal complementary sensitivity function. The control-system bandwidth: is 0.06 .

4 - You compute the parameters $a \mathrm{k}, b \mathrm{k}, v \mathrm{r}, v \mathrm{k}$ an $\mathrm{d} v \mathrm{k}+1$ in order to obtain the optimal open-loop Nichols locus (figure 12). Using the CRONE Control Toolbox, you obtain:

$a_{-1}=1 ; b_{-1}=0 ; 4 ; a_{0}=-21.32, b_{0}=-16.6 ; a_{1}=1$;

$b_{1}=2.5 ; v_{r}=0,065 ; v_{-1}=0,0005 ; v_{0}=0,001$;

$v_{1}=0,3 ; v_{2}=0,7$.

5 - Finally, you synthesize the controller transfer function:

$$
K(w)=1.15 \frac{(w+0.25)(w+0.03)\left(w^{2}+3.6 e^{-4} w+2.025 e^{-5}\right)}{(w+3.5)(w+1)^{2}\left(w+5 e^{-3}\right) w^{2}}
$$

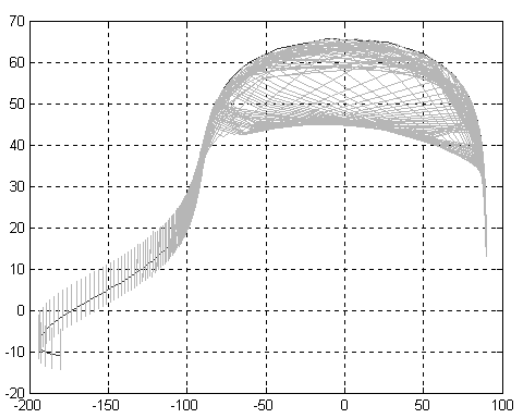

Figure 11 - Nominal open-loop transfer function and uncertainty domains

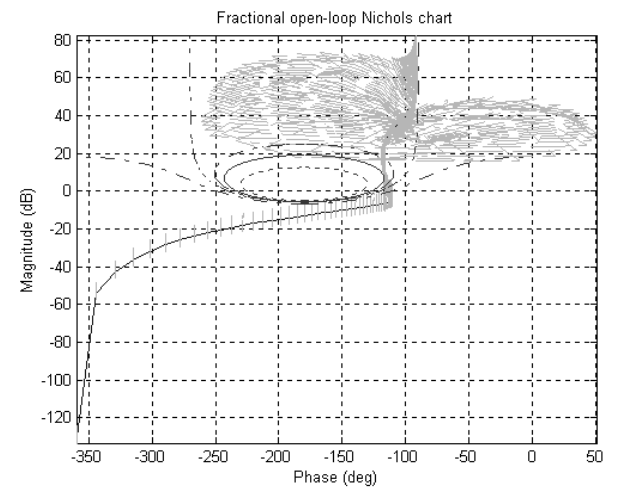

Figure 12 - Optimal open-loop Nichols locus

\section{4 - Comparison of the two methodologies}

\subsection{1 - $\mu$-analysis results}

The first comparison is achieved using a $\mu$-analysis that is a powerful tool to analyze the robustness properties of linear feedback systems. $\mu_{\Delta}$ is the structured singular value related to an uncertainty structure $\Delta$. A more accurate definition can be found in $[1,2,4] . \mu$-analysis allows to achieve a structured analysis of the robustness properties of a feedback system and to find the admissible interval for each parameter. Thus results are less conservative than with an unstructured analysis made simply with the norm $\mathrm{H}$-infinity. 
Theorem (Generalization of small gain theorem):

Let $\beta>0$. The loop in figure 2 is internally stable for all $\Delta$ with $\|\Delta\|_{\infty}<\beta^{-1}$ if and only if: $\sup _{\omega} \mu_{\Delta}\left(T_{z w}(j \omega)\right) \leq \beta$.

This theorem is used in order to study the modulus margin robustness of the two feedback systems defined in sections 4.1 and 4.2. We consider a mixed (real and complex) $\Delta$ in order to avoid discontinuity problem.

Results for the H-infinity design: $\max \mu_{\Delta}=0.98$

thus modulus margin robustness is warranted for $888<K_{s}<12112 \mathrm{~N} / \mathrm{m}$ and $1.6<M_{s}<84.75 \mathrm{Kg}$.

Results for the CRONE design: $\max \mu_{\Delta}=0.89$

thus modulus margin robustness is warranted for $320<K_{s}<12680 \mathrm{~N} / \mathrm{m}$ and $0.45<M_{s}<102 \mathrm{Kg}$.

So the results are good for both controllers since they guaranty the modulus margin robustness for the parameter variations given in section 4.1 .

\subsection{2 - Simulation results}

The figure 13 shows the simulation results for a given trajectory and the nominal, minimal and maximal load with both controllers. The CRONE controller is more robust to parametric variation. It leads to shorter settling times than $\mathrm{H}-$ infinity controller although the weighting function $W_{1}$ has been chosen to get the larger possible bandwidth.

\section{5 - Conclusion}

Two robust control-system design methodologies have been studied and compared in this article: the H-infinity control system design and the CRONE control system design.

The interest of the H-infinity control-system design is the state-space representation that allows easy computation for MIMO systems, even if the case of these systems has not been studied in this article. The drawbacks are the conservative results if you consider unstructured uncertainty defined with the $\mathrm{H}$-infinity norm since plant perturbation are then over-estimated. If you want to limit this conservatism, you have to design the $\mathrm{H}$-infinity controller while forgetting the plant uncertainty and then you have to make a $\mu$-analysis to check the robustness of the controller. But then robustness has to be checked after the controller design. If results are not good, you have to re-run the computation with other weighting functions. Otherwise, you can do a $\mu$-synthesis but this is another methodology and other drawbacks exist.

The interests of CRONE control-system design are multiple. The use of complex fractional differentiation permits to define the open-loop transfer function with few high-level parameters. The optimization problem that leads to the optimal transfer function to meet the specifications is thus easier to solve. Moreover, Crone control design takes into account the genuine plant perturbation without overestimation, then better performance can be obtained. Now CRONE control system design has to extend its applications. CRONE control has already been applied to squared MIMO systems but not yet not to non squared MIMO systems.


H-infinity controller
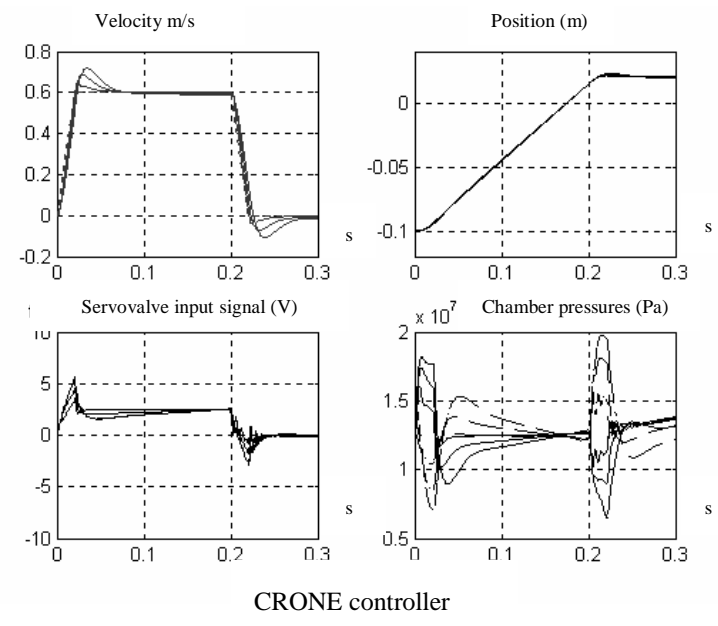

Figure 13 - Simulation results

\section{Bibliography:}

[1] D. Alazard, et al, (1999), «Robustesse et commande optimale », Editions Cepadues, Toulouse

[2] G. Duc, S. Font, (1999), «Commande Hinfini et $\mu$ analyse », Editions Hermès, Paris

[3] K. Glover, D. MCFarlane, (1989), «Robust Stabilization of normalized coprime factor plant description with H-infinity bounded uncertainty », IEEE. Trans. Autom. Control, AC 34 n 8 pp 821-830

[4] W.S. Levine (1996), «The Control Handbook», CRC Press \& IEEE Press

[5] Mathworks « Robust control Toolbox», User's guide

[6] A. Oustaloup, J. Sabatier and P. Lanusse (1999), «From fractal robustness to the Crone control», Fractional Calculus and Applied Analysis, Vol. 2, n 1 , 1999

[7] A.Oustaloup, B.Mathieu (1999), «La commande CRONE du scalaire au multivariable », Hermès, Paris 10

[8] A.Oustaloup, et al, (2000), «The CRONE toolbox for Matlab», 11th IEEE International Symposium on Computer-Aided Control System Design, CACSD Anchorage, Alaska, USA, September 25-27 